\title{
CANCER
}

\section{Net closes in on genetics of liver fluke-associated cholangiocarcinoma}

Findings published in Nature Genetics provide the first insights into the mutational landscape of liver fluke-related cholangiocarcinoma (CCA).

"CCA associated with infection by Opisthorchis viverrini is a major health problem in northeastern Thailand, Laos and Cambodia, but not much is known about the genetic basis of this cancer," explain Bin Tean Teh and Patrick Tan, co-senior authors.

Teh and colleagues performed wholeexome sequencing of eight $O$. viverriniassociated CCAs and matched normal tissue. 206 somatic mutations in 187 genes were identified and validated by Sanger sequencing. 13 genes with recurrent somatic mutations (in $\geq 2$ tumours) were selected and their mutation frequency determined in a prevalence set of 46 O. viverrini-related CCA cases. CDKN2A and PTEN were also included to enable comparison with data from hepatocellular carcinoma (HCC) and pancreatic ductal adenocarcinoma (PDAC) studies.

The $O$. viverrini-associated CCAs were found to contain nonsynonymous (aminoacid altering) somatic mutations in known CCA-related genes, such as TP53 (44.4\% of samples), and newly implicated genes, such as KRAS (16.7\%), SMAD4 (16.7\%) and MLL3 (14.8\%). "The mutations in $M L L 3$, which encodes a histone modifier, seem to be independent of TP53, KRAS and SMAD4 mutations, indicating the importance of $M L L 3$ in this cancer," clarifies Teh. The importance of genome stability and G-protein signalling in CCA was confirmed by the other genes identified (GNAS, ROBO2, RNF43, PEG3, XIRP2, RADIL, NDC80, PCDHA13 and LAMA2).

In addition, although some of the alterations detected were unique to CCA, the authors suggest that the mutational landscape of CCA has more in common with that of PDAC than that of HCC.

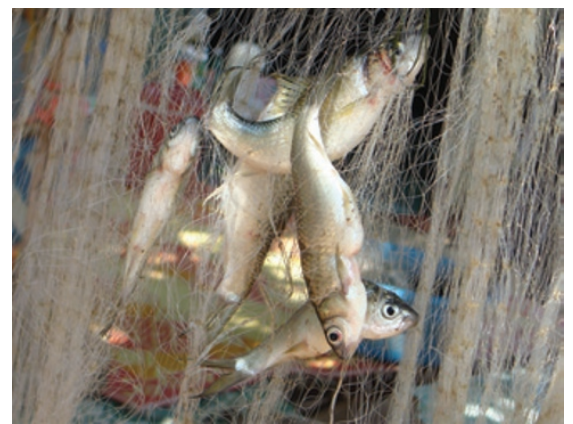

Cyprinoid fish are an intermediate host for the Opisthorchis viverrini liver fluke. Courtesy of B.T. Teh.

So, what's next for the researchers? "We plan to start further functional studies, including generating mouse models of this cancer, to demonstrate the importance or transforming potential of the mutated genes that we identified," says Teh. "Our findings provide tremendous new opportunities for research on this cancer."

Natalie J. Wood

Original article Ong, C. K. et al. Exome sequencing of liver fluke-associated cholangiocarcinoma. Nat. Genet. doi:10.1038/ng.2273 